\title{
The Cosmic Infrared Background Experiment
}

\author{
James Bock ${ }^{1,2}$, John Battle ${ }^{1}$, Asantha Cooray ${ }^{3}$, Mitsunobu Kawada ${ }^{4}$ \\ Brian Keating ${ }^{5}$, Andrew Lange ${ }^{2}$, Dae-Hea Lee $^{6}$, Toshio Matsumoto ${ }^{7}$ \\ Shuji Matsuura ${ }^{7}$, Soojong Pak ${ }^{6}$, Tom Renbarger ${ }^{5}$, Ian Sullivan ${ }^{2}$ \\ Kohji Tsumura ${ }^{7}$, Takehiko Wada ${ }^{7}$, Toyoki Watabe ${ }^{4}$ \\ ${ }^{1}$ Jet Propulsion Laboratory, Pasadena CA 91109 \\ ${ }^{2}$ California Institute of Technology, Pasadena CA 91125 \\ ${ }^{3}$ Physics and Astronomy, UC Irvine, Irvine CA 92697 \\ ${ }^{4}$ Nagoya University, Furo-cho, Chikusa-ku, Nagoya 464-8602, Japan \\ ${ }^{5}$ Department of Physics, UC San Diego, San Diego CA 92093 \\ ${ }^{6}$ Korea Astronomy and Space Science Institute, Daejeon 305-348, Korea \\ ${ }^{7}$ ISAS, JAXA, Sagamihara, Kanagawa 229-8510, Japan \\ E-mail: James.Bock@jpl.nasa.gov
}

\begin{abstract}
The extragalactic background, based on absolute measurements reported by DIRBE and IRTS at 1.2 and $2.2 \mu \mathrm{m}$, exceeds the brightness derived from galaxy counts by up to a factor 5. Furthermore, both DIRBE and the IRTS report fluctuations in the near-infrared sky brightness that appear to have an extra-galactic origin, but are larger than expected from local $(\mathrm{z}=1-3)$ galaxies. These observations have led to speculation that a new class of high-mass stars or mini-quasars may dominate primordial star formation at high-redshift $(z \sim 10-20)$, which, in order to explain the excess in the near-infrared background, must be highly luminous but produce a limited amount of metals and X-ray photons. Regardless of the nature of the sources, if a significant component of the near-infrared background comes from first-light galaxies, theoretical models generically predict a prominent near-infrared spectral feature from the redshifted Lyman cutoff, and a distinctive fluctuation power spectrum.
\end{abstract}

We are developing a rocket-borne instrument (the Cosmic Infrared Background ExpeRiment, or CIBER) to search for signatures of primordial galaxy formation in the cosmic near-infrared extra-galactic background. CIBER consists of a widefield two-color camera, a low-resolution absolute spectrometer, and a high-resolution narrow-band imaging spectrometer.

The cameras will search for spatial fluctuations in the background on angular scales from $7^{\prime \prime}$ to $2^{\circ}$, where a first-light galaxy signature is expected to peak, over a range of angular scales poorly covered by previous experiments. CIBER will determine if the fluctuations reported by the IRTS arise from first-light galaxies or have a local origin. In a short rocket flight CIBER has sensitivity to probe fluctuations 
$100 \times$ fainter than IRTS/DIRBE, with sufficient resolution to remove local-galaxy correlations. By jointly observing regions of the sky studied by Spitzer and ASTROF, CIBER will build a multi-color view of the near-infrared background, accurately assessing the contribution of local $(\mathrm{z}=1-3)$ galaxies to the observed background fluctuations, allowing a deep and comprehensive survey for first-light galaxy background fluctuations.

The low-resolution spectrometer will search for a redshifted Lyman cutoff feature between $0.8-2.0 \mu \mathrm{m}$. The high-resolution spectrometer will trace zodiacal light using the intensity of scattered Fraunhofer lines, providing an independent measurement of the zodiacal emission and a new check of DIRBE zodiacal dust models. The combination will systematically search for the infrared excess background light reported in near-infrared DIRBE/IRTS data, compared with the small excess reported at optical wavelengths.

Key words: extragalactic background, primordial galaxies, infrared

\section{The Infrared Background}

Is the infrared background (IRB) entirely produced by discrete local galaxies, or is there an unaccounted component? Integrated individual galaxy counts appear to fall short of the IRB measured with absolute photometry at nearinfrared wavelengths. The cause is either unaccounted systematic errors, or a tantalizing clue to new cosmology: a diffuse IRB component from the early universe. A new population of energetic sources at high redshift (1), (2), (3) would be needed to explain the intensity of the near-infrared background as reported by several authors. Because the infrared background may have profound implications for cosmology, these measurements must be confirmed by independent means.

The IRB may be studied with absolute photometry or integrated number counts of galaxies. Both methods are confronted with systematic errors. With absolute photometry, one must account for instrument emission and baselines, and remove local foregrounds, namely stars, interstellar dust, interplanetary dust emission, at mid-infrared and far-infrared wavelengths, and sunlight scattered by interplanetary dust ('zodiacal light'), at optical and near-infrared wavelengths (4). Galaxy counts could possibly under-count flux from diffuse components or miss objects with low surface brightness, especially in groundbased infrared surveys using spatial chopping (see (5) for a quantitative assessment).

We compile recent measurements of the extragalactic near-infrared/optical background in Figure 1 based on DIRBE (6), (7), (8), (9), (10), (11), IRTS (12), HST (13), and optical measurements (14), (15) for absolute photometery, 
and integrated galaxy number counts $(\underline{5}),(16),(17)$.

In the near-infrared and optical bands, the dominant foreground contaminant is zodiacal light. The IRTS and many DIRBE-based results use a model of IPD scattering and emission (18). Wright 2001 and Gorjian et al. (2000) use a different model based on the principle that the zodiacal residual be zero at 25 $\mu m$ at high ecliptic latitude (19). The optical measurement of Bernstein et al. 2002 subtracts zodiacal emission based on the observed strength of scattered Fraunhofer lines from a ground-based measurement (20). Unfortunately, zodiacal dust models are not unique and represent a leading source of systematic error.

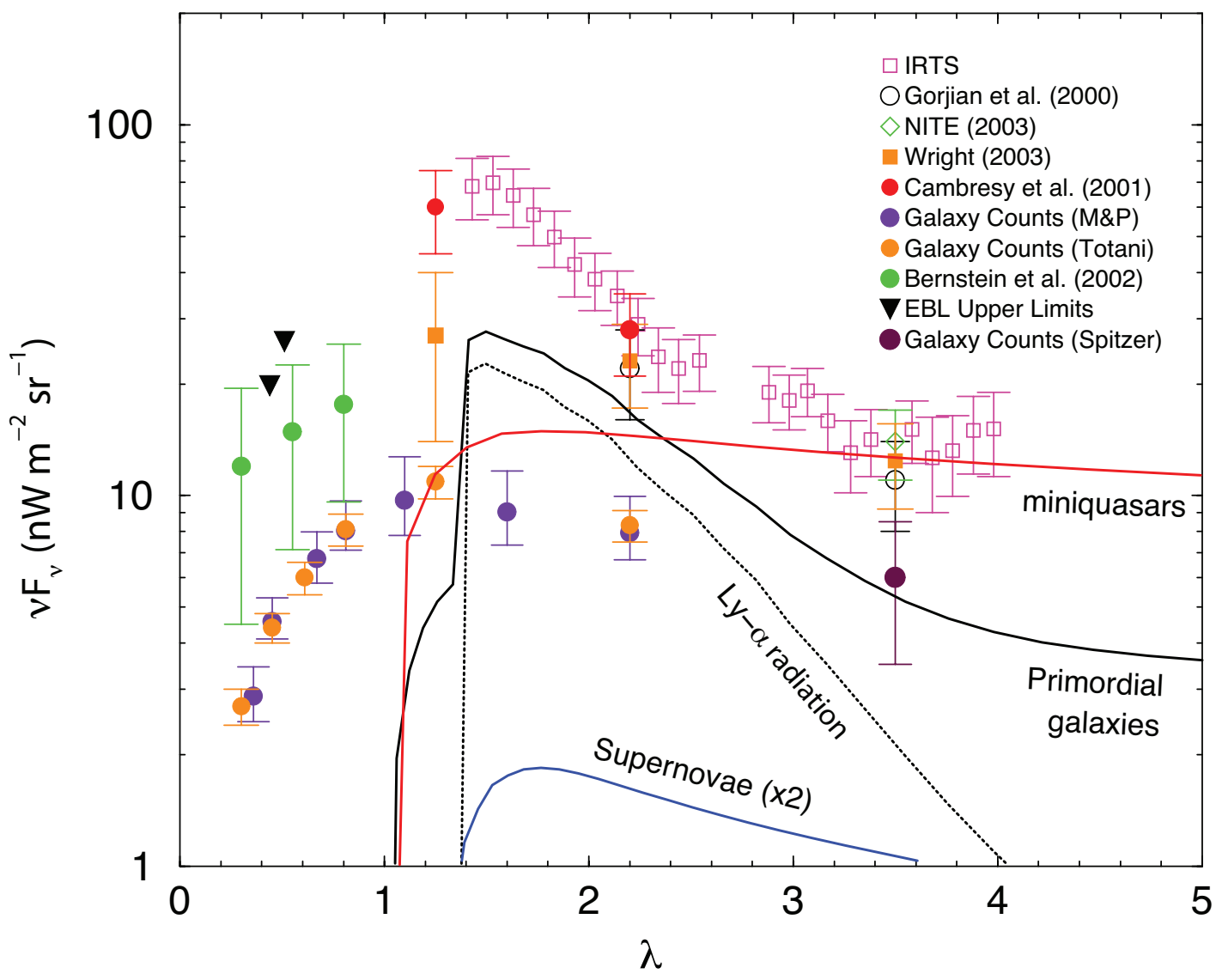

Fig. 1. Summary of observations of the extragalactic background at near-infrared and optical wavelengths, showing upper limits, reported residuals after subtraction of local foregrounds but without proof of isotropy (open symbols), reported detections, and integrated galaxy counts. The large experimental scatter at $1.2 \mu \mathrm{m}$ is notable, the choice of zodiacal model being a prime culprit for systematic error. The curves indicate model FLG-IRB components arising from ionizing radiation sources at $z \sim 10-20$, including population III star formation in primordial galaxies, mini-quasars, and supernovae

Nevertheless, Figure 1 shows an intriguing trend: the integrated galaxy counts appear to fall well short of the IRB measured with absolute photometry in the near-infrared (particularly in the 1.2 and $2.2 \mu \mathrm{m}$ DIRBE bands), but are 
marginally consistent with the HST data at optical wavelengths, and with Spitzer at $3.6 \mu \mathrm{m}$. Furthermore, the absolute photometry measurements appear to show a broad maximum at 1-2 $\mu \mathrm{m}$. This peak is either pronounced (the IRTS spectrum), or modest (the Wright data points). Clearly one must be duly cautious of systematic errors. However, the data may be hinting at the existence of a low-surface brightness component with a spectrum peaked at 1-2 $\mu \mathrm{m}$, which is the generic Ly-cutoff signature one expects from first-light galaxies at high redshift.

\section{The Cosmic Infrared Background ExpeRiment}

DIRBE and the IRTS have carried out a comprehensive measurement of the infrared sky brightness with absolute photometry, in the case of DIRBE involving highly redundant all-sky measurements. Nevertheless, key attributes of the IRB remain unexplored, and can be addressed in a short sounding rocket experiment, needed to escape strong airglow emission (21), (22). CIBER will search for a first-light component to the IRB by studying the spatial anisotropy of the IRB on angular scales from $7^{\prime \prime}$ to $2^{\circ}$. A fluctuations experiment has to potential to probe for a first-light galaxy (FLG) IRB component to a level roughly an order of magnitude fainter (23), (24) than has been realized with absolute photometry. CIBER will study the absolute spectrum of the IRB in the uncovered region between HST at $0.8 \mu \mathrm{m}$ and IRTS and DIRBE at $1.2 \mu \mathrm{m}$. Finally, CIBER will measure the intensity of the zodiacal foreground using near-infrared Fraunhofer lines, allowing an independent check of the Kelsall et al. zodiacal dust model.

\section{Wide-Field Imagers}

CIBER's imager optics are designed to detect FLG-IRB fluctuations from high redshift $(z \sim 10-20)$ by producing deep, wide-field images on spatial scales from $7^{\prime \prime}$ to $2^{\circ}$. Fluctuations from foregrounds (e.g. zodiacal light, galaxies) can be discriminated by using spatial spectra from the two bands (see Figure 2). The imager data will be combined with shallow Spitzer and ASTRO-F survey fields to allow us to detect and remove galaxies to a deeper level than possible with CIBER alone. Spitzer and ASTRO-F will make significant contributions in this field (see (25)); however, CIBER images at shorter wavelengths, which is especially important if the fluctuating component shows a redshift-integrated Ly-cutoff signature. Furthermore, CIBER is a wide-field instrument optimized to detect FLG-IRB fluctuations on large angular scales, with the wide multipole coverage needed to discriminate local-galaxy fluctuations. By observing the same fields as ASTRO-F and Spitzer, CIBER provides a powerful check of systematic artifacts (e.g. flat fielding and mosaicing) between instruments, and the combination gives comprehensive multi-color information. 
The wide-field camera is a 10-cm refracting telescope cooled to $77 \mathrm{~K}$, imaging onto a $1024^{2} \mathrm{HgCdTe}$ focal plane array and providing $7^{\prime \prime}$ pixels and a $2^{\circ} \times$ $2^{\circ}$ field of view. The detector array, sampled every $1.7 \mathrm{~s}$ with a $25 \mathrm{~s}$ reset interval, provides background-limited performance. A cold shutter allows us to record dark frames prior to launch, and on descent after observations have completed. In the limited observation time available on a sounding rocket flight, the cameras provide high sensitivity to diffuse emission, with fidelity to signals on large angular scales. Note that the sensitivity shown in Table 1 and Figure 2 is for only a single 50 s field, and we will have six such fields in a flight.

Table 1: Imager Specifications

\begin{tabular}{|l|c|c|l|}
\hline Aperture & \multicolumn{2}{|c|}{11} & cm \\
Pixel size & \multicolumn{2}{|c|}{$7 \times 7$} & arcsec \\
FOV & \multicolumn{2}{|c|}{$2 \times 2$} & degrees \\
\hline$\lambda$ & $0.95(\mathrm{I})$ & $1.6(\mathrm{H})$ & $\mu m$ \\
$\Delta \lambda / \lambda$ & 0.5 & 0.5 & \\
Array QE & 0.65 & 0.75 & \\
Optics QE & 0.85 & 0.85 & \\
Photo current & 12 & 11 & $e^{-} / s$ \\
Dark current & $<0.3$ & $<0.3$ & $e^{-} / s$ \\
Read noise (CDS) & 15 & 15 & $e^{-}$ \\
$\delta \nu I_{\nu}($ inst $)$ & $39(1 \sigma)$ & $19(1 \sigma)$ & $n W m^{-2} s^{-1} p^{-1} x^{-1}$ \\
\hline CIBER source cut & $18.6(3 \sigma)$ & $18.0(3 \sigma)$ & vega mag \\
& $2.2 \mathrm{e} 3$ & $4.5 \mathrm{e} 3$ & sources / sq degree \\
\hline Deep galaxy cut & 23.0 & 21.4 & vega mag \\
& $6 \mathrm{e} 4$ & $6 \mathrm{e} 4$ & sources / sq degree \\
\hline
\end{tabular}

sensitivities quoted for a 50 s observation

CIBER reaches a limiting point source sensitivity of $\mathrm{I}=18.5$ and $\mathrm{H}=17.8 \mathrm{mag}$ $(3 \sigma)$, somewhat deeper than the 2MASS all-sky survey. Source confusion (16) is 2-3 times fainter than the instrument noise level, assuming that pixels are clipped at $3 \sigma$. In order to remove the local-galaxy clustering foreground more completely, deeper removal of point sources will be necessary. The pixel size of $7^{\prime \prime}$ is chosen to give us a sufficient density of pixels to remove sources down to $\mathrm{I}=23, \mathrm{H}=21.5$ using ancillary ground-based data. CIBER pixels may be simply masked down to these cutoff magnitudes with acceptable $(\sim 25 \%)$ data loss. 


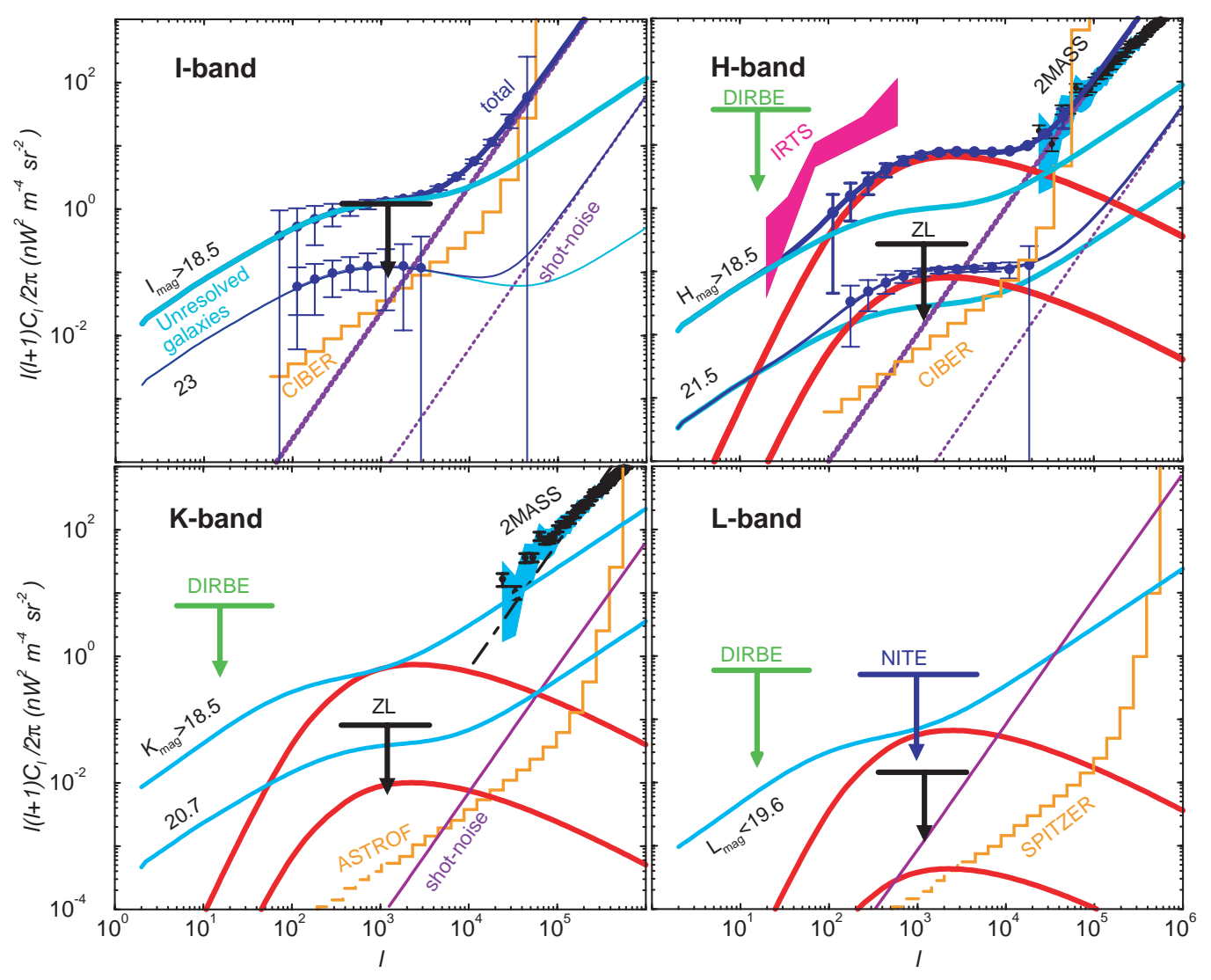

Fig. 2. Spatial power spectrum of the IRB in standard IR bands. The red curves peaking at $l=1000$ show the power spectra of first-light galaxy fluctuations from $10<z<20$, with optimistic (top line) and pessimistic (bottom line) estimates. The cyan curves give foreground galaxy clustering, the dotted lines show foreground galaxy shot noise, as a function of magnitude cutoff. The blue data points show the sensitivity of CIBER on a single observation field, in multipole bins, including both statistical noise and sample variance, on the optimistic and pessimistic models, using moderate and deep source cutoffs. The orange stair-steps show the statistical noise in log multipole bins. The results of previous experiments are indicated for DIRBE (27), 2MASS (28), IRTS (12), and NITE (8). The orange stair-steps, including foregrounds from their $3 \sigma$ point source cutoffs, show the statistical sensitivities of Spitzer and ASTRO-F in their shallow survey fields, with dashed lines showing where field mosaicing is needed. Upper limits on zodiacal fluctuations are shown, scaling from data with a $3^{\prime}$ beam at $25 \mu \mathrm{m}(29)$ by the zodiacal spectrum.

CIBER has the sensitivity to detect even the fluctuations from the pessimistic first-light galaxy model. We note that the optimistic first-light galaxy model assumes highly biased formation and contributes $\nu I_{\nu}=25 \mathrm{nWm}^{-2} \mathrm{sr}^{-1}$ to the IRB; the pessimistic model assumes moderately biased formation and contributes $\nu I_{\nu}=3 n W^{-2} s r^{-1}$. Thus a comprehensive fluctuations measurement can probe for a first-light galaxy component to very low levels, well below the current state of controversy in the IRB measurements (and see (26)).

\section{Low-Resolution Spectrometer}


The low-resolution spectrometer is designed to measure the absolute spectrum of the near-infrared background in the 0.8-2.0 $\mu \mathrm{m}$ spectral region, in a search for a redshifted Ly-cutoff feature from first-light galaxies. As shown in Figure 1, the IRB may peak at 1-2 $\mu \mathrm{m}$, and fall in brightness in the optical. The transition from the near-infrared to the optical, if present, would have a much different spectral shape than either zodiacal light or the integrated counts of galaxies, and could be detected without precise removal of the zodiacal foreground.

The spectrometer is a $7.3 \mathrm{~cm}$ refracting telescope with re-imaging optics. A prism inserted in the collimated beam produces low-resolution spectra $(R \sim$ $20, \lambda=0.8-2.0 \mu m)$. We use a $256^{2} \mathrm{HgCdTe}$ array with a pixel size of 40 $\mu m$, producing $1^{\prime} \times 1^{\prime}$ pixels and total field of view of $4^{\circ}$. Four slits located at the field stop produce $1^{\prime} \times 4^{\circ}$ strips on the sky, which the prism disperses into separate spectra on the array. As a result, 256 spectra are available for each slit, and a total of 1024 spectra are obtained.

\section{Table 2: Low-Resolution Spectrometer Specifications}

\begin{tabular}{|l|c|l|}
\hline Aperture & 7.3 & $\mathrm{~cm}$ \\
Pixel size & $1 \times 1$ & arcmin \\
FOV & $4 \times 4$ & degrees \\
Wavelength range & $0.8-2.0$ & $\mu m$ \\
Resolution & $\Delta / \Delta \lambda=21-23$ & \\
Slit size & $1 \times 256$ & arcmin \\
Optics QE & 0.8 & \\
Array QE & 0.5 & \\
Photo current & $10 \sim 20$ & $e^{-} / s$ \\
Dark current & $<0.1$ & $e^{-} / s$ \\
Read noise $(\mathrm{CDS})$ & $<30$ & $e^{-}$ \\
\hline
\end{tabular}

The expected sensitivity of the spectrometer, which achieves backgroundlimited performance, is shown in Figure 3 together with the zodiacal sky brightness, and the IRB residual brightness and fluctuation spectra reported by the IRTS. The specifications of the spectrometer are summarized in Table 2 . In order to obtain an accurate spectrum of the sky, we may co-add independent spectra. After rejecting spectra that include point sources brighter than $18^{\text {th }}$ mag using the imager data, 400 such spectra can be co-added. The resulting sensitivity allows us to detect the absolute sky brightness with $\mathrm{S} / \mathrm{N}$ $>100$ in only $15 \mathrm{~s}$ of integration time. By co-adding four spatial and four spectral pixels, we can detect IRTS/DIRBE fluctuations with $S / N \sim 5$, and we anticipate having 300 such spatially independent pixels to this sensitivity. 


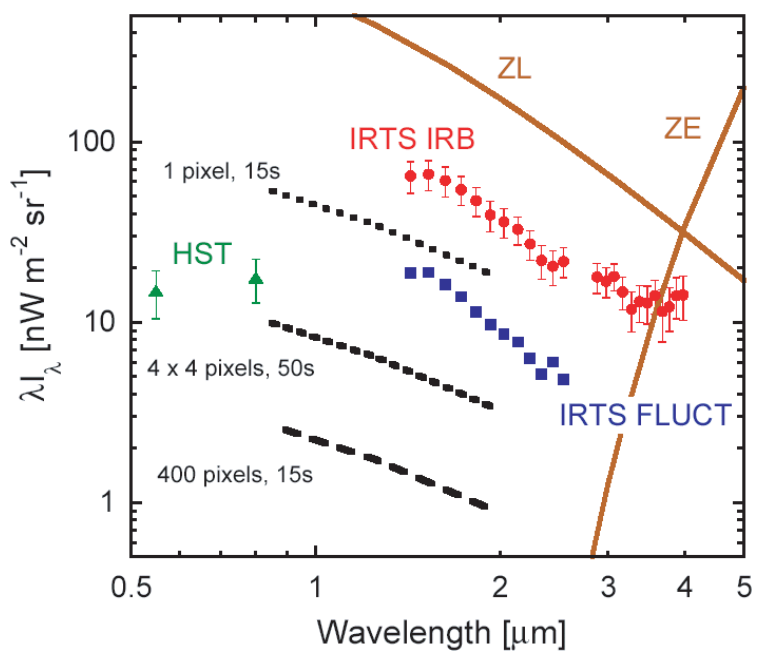

Fig. 3. Detection limits of the spectrometer at $1 \sigma$ significance (dashed lines) for a single pixel, co-addition of 4 spatial and 4 spectral pixels, and co-addition of 400 spatial pixels (the entire field of view expected to be free from point sources). Shown in red and blue data points are the IRB and RMS fluctuations reported by the IRTS. The zodiacal light spectrum at the ecliptic pole is shown for reference.

\section{High-Resolution Spectrometer}

Uncertainties in zodiacal foreground removal play a leading role in the systematic errors in determining the IRB, as evident by the large dispersion of data points at $1.2 \mu \mathrm{m}$ in Figure 1, which are the result of using different zodiacal dust models based on DIRBE observations.

Prominent features in the solar spectrum can be used to trace the zodiacal light since it is dominated by scattered sunlight at these wavelengths. Narrowband spectroscopy, centered on and just off a Fraunhofer line, has been used to estimate and remove the zodiacal light for optical extragalactic background measurements (13), (14), and to study the dynamics of the zodiacal dust cloud (30). While simple in concept, this technique is prone to its own set of systematic errors (31), most notably absolute calibration, atmospheric extinction, atmospheric scattering of zodiacal light and Galactic starlight, and even atmospheric scattering of reflections off the ground. Airglow emission greatly complicates measurements at $\lambda>0.55 \mu \mathrm{m}$ from the ground.

With a small cooled spectrometer on a sounding rocket, we can measure nearIR Fraunhofer features for direct comparison to the DIRBE zodiacal model, avoiding the systematic errors associated with atmospheric scattering, and can absolutely calibrate the instrument with a beam-filling source in the lab.

The narrow-band spectrometer is a $7.5 \mathrm{~cm}$ imaging refractor with a narrowband filter placed as the first optical element. Rays passing through such a dielectric etalon experience a wavelength shift $\lambda=\lambda_{0} \cos \theta_{i}$, where $\theta_{i}$ is the 
angle of the ray inside the dielectric. Thus the wavelength response of the spectrometer varies over the array, by an amount depending of the field of view. This can be tailored so that the response at the array center is tuned on the absorption feature, and shifts entirely off the feature at the edge of the array.

Table 3: High-Resolution Spectrometer Specifications

\begin{tabular}{|c|c|c|c|}
\hline Aperture & \multicolumn{2}{|c|}{7.5} & $\mathrm{~cm}$ \\
\hline Pixel size & \multicolumn{2}{|c|}{$2 \times 2$} & $\operatorname{arcmin}$ \\
\hline FOV & \multicolumn{2}{|c|}{$8.5 \times 8.5$} & degrees \\
\hline Resolution & \multicolumn{2}{|c|}{1000} & \\
\hline Filter QE & \multicolumn{2}{|c|}{0.7} & \\
\hline Optics QE & \multicolumn{2}{|c|}{0.9} & \\
\hline Array QE & \multicolumn{2}{|c|}{0.65} & \\
\hline Photo current & \multicolumn{2}{|c|}{1.5} & $e^{-/ s}$ \\
\hline Dark current & \multicolumn{2}{|c|}{$<0.1$} & $e^{-} / s$ \\
\hline Read noise (CDS) & \multicolumn{2}{|c|}{20} & $e^{-}$ \\
\hline Wavelength & 0.8542 & 1.069 & $\mu m$ \\
\hline Line strength & 0.27 & 0.07 & $\mathrm{~nm}$ \\
\hline Contrast & 25 & 6 & $\%$ \\
\hline$\nu I_{\nu}($ sky at $\mathrm{NEP})$ & 550 & 450 & $n W m^{-2} s r^{-1}$ \\
\hline$\delta \nu I_{\nu}$ & $55(1 \sigma)$ & $60(1 \sigma)$ & $n W m^{-2} s r^{-1} p i x^{-1}$ \\
\hline $\mathrm{S} / \mathrm{N}$ zodi & 170 & 40 & $100 \times 100$ pix \\
\hline$\Delta \nu I_{\nu}$ (zodiacal zero point) & $3(1 \sigma)$ & $12(1 \sigma)$ & $n W m^{-2} s r^{-1}$ \\
\hline
\end{tabular}

sensitivities quoted for a $50 \mathrm{~s}$ observation

As shown in Table 3, the spectrometer can infer the zodiacal intensity with $S / N=40$ observing a collection of lines at $1.07 \mu \mathrm{m}$, or $S / N=170$ observing the $0.85 \mu \mathrm{m}$ CaII line, at the north ecliptic pole in a $50 \mathrm{~s}$ observation (and with higher $\mathrm{S} / \mathrm{N}$ at lower ecliptic latitudes). The photon level, signal contrast, and the $S / N$ per pixel are low, so careful attention must be paid to dark current, stray light, and out-of-band blocking. We will accurately measure the dark current in flight using a cold shutter.

To compare with the Kelsall et al. zodiacal dust model, we must use the measured zodiacal color from the low-resolution spectrometer to extrapolate the zodiacal signal to the 1.2 and $2.2 \mu \mathrm{m}$ DIRBE bands. The low-resolution spectrometer data are essential because the zodiacal spectrum departs from 
the solar spectrum around $1 \mu m$, and has not been accurately measured at $\lambda<1.2 \mu \mathrm{m}$.

Finally we note the Fraunhofer line measurements provide an absolute tracer of the zodiacal foreground, and combined with the low-resolution spectrometer, allow a determination of the absolute infrared background from $0.8-2.0 \mu \mathrm{m}$.

\section{Summary}

Because CIBER has multiple capabilities, we summarize the measurement objectives. CIBER will:

- Probe the IRTS fluctuations in several fields with $100 \times$ higher signal-tonoise, using spatial and color information to determine if the IRTS fluctuations arise from zodiacal, Galactic, local-galaxy clustering, or first-light galaxy origins.

- Use CIBER's $100 \times$ higher sensitivity, 100× higher angular resolution, and $500 \times$ deeper point source removal than IRTS to probe the spectrum of IRB fluctuations for a FLG-IRB component at a level $100 \times$ fainter than the IRTS fluctuations. Combine CIBER data with ASTRO-F and Spitzer surveys for the definitive data set for IRB fluctuations in 4 bands from 0.9 to $3.6 \mu \mathrm{m}$.

- Search for the Lyman cutoff signature of reionization in the IRB between the optical and near-infrared IRB measurements.

- Measure the zodiacal foreground in several fields using a narrow-band spectrometer to trace scattered near-infrared Fraunhofer absorption features. Current zodiacal model discrepancies based on DIRBE are at the $\sim 10 \%$ level, producing large discrepancies in the derived IRB.

- Combine the low-resolution and narrow-band spectrometer data to probe the IRB from $0.8-2.0 \mu m$ down to the narrow-band spectrometer's zodiacal subtraction limit.

The authors wish to acknowledge the support of NASA grant NNG05WC18G.

\section{References}

[1] S. Wyithe \& A. Loeb, Ap. J. 588, L69 (2003).

[2] A. Cooray \& N. Yoshida, MNRAS 51, L71 (2004) astro-ph/0404109

[3] P. Madau \& J. Silk, MNRAS 359, 37 (2005).

[4] M. G. Hauser \& E. Dwek, ARAA 39, 249 (2001).

[5] T. Totani et al., Ap. J. 550, L137 (2001).

[6] E. L. Wright, Ap. J. 553, 538 (2001). 
[7] L. Cambresy et al., Ap. J. 555, 563 (2001).

[8] J. Xu et al., Ap. J. 580, 653 (2002).

[9] V. Gorjian, E. L. Wright \& R. R. Chary, Ap. J. 536, 550 (2000).

[10] E. Dwek \& R. G. Arendt, Ap. J. 508, 9 (1998).

[11] M. G. Hauser et al., Ap. J. 508, 25 (1998).

[12] T. Matsumoto et al., Ap. J. 626, 31 (2005).

[13] R. A. Bernstein, W. L. Freedman \& B. F. Madore, Ap. J. 571, 56 (2002).

[14] R. R. Dube, W. C. Wickes and D. T. Wilkinson, Ap. J. 232, 333 (1979).

[15] G. N. Toller, Ap. J. 266, L79 (1983).

[16] P. Madau \& L. Pozzetti, MNRAS 312, L9 (2000) and references therein.

[17] G. G. Fazio et al., Ap. J. Supp. 154, 39 (2004).

[18] T. Kelsall et al., Ap. J. 508, 44 (1998).

[19] E. L. Wright, MNRAS 29, 1354 (1997).

[20] R. A. Bernstein, W. L. Freedman \& B. F. Madore, Ap. J. 571, 85 (2002).

[21] S. K. Ramsay, C. M. Mountain \& T. R. Geballe, MNRAS 259, 751 (1992).

[22] www.pegasus.phast.umass.edu/2mass/teaminfo/airglow.html

[23] A. Cooray et al., Ap. J. 606, 611 (2004) astro-ph/0308407

[24] A. Kashlinsky et al., Ap. J. 608, 1 (2004).

[25] A. Kashlinsky et al., these proceedings (2005).

[26] F. Aharonian et al., astro-ph 0508073 (2005).

[27] A. Kashlinsky \& S. Odenwald, Ap. J. 528, 74 (2000).

[28] A. Kashlinsky et al., Ap. J. 579, 53 (2002).

[29] P. Abraham, C. Leinert \& D. Lemke, A. \& A. 328, 702 (1997).

[30] R. J. Reynolds, G. J. Madsen \& S. H. Moseley, Ap. J. 612, 1206 (2004).

[31] K. Mattila, Ap. J. 591, 119 (2003). 